\title{
THE DYNAMICS OF SLIM DISKS WITH LARGE-SCALE MAGNETIC FIELDS*
}

\author{
RENYI MA \\ Department of Astronomy and Institute of Theoretical Physics and Astrophysics, \\ Xiamen University, Xiamen 361005, Fujian Province, China \\ ryma@xmu.edu.cn
}

Received 31 October 2013

Revised 29 November 2013

Published 21 March 2014

\begin{abstract}
As suggested by recent observations, we explore the influences of large-scale magnetic fields on the dynamics of slim disks. The magnetic fields are assumed to be self-similar and be a fixed fraction of the total pressure at the footpoint. The global solutions show that the radial velocity increases and the disk temperature decreases with enhancing magnetic fields. The ratio of the jet kinetic power to disk luminosity is less than 0.1, which indirectly support the argument that radio-loud (RL) narrow-line Seyfert 1 galaxies (NLS1s) are similar to blazars, with jets pointing to us.
\end{abstract}

Keywords: Accretion and accretion disks; jets; magnetic fields.

PACS Numbers: $98.62 . \mathrm{Mw}, 98.62 . \mathrm{Nx}, 83.60 . \mathrm{Np}$

\section{Introduction}

Radio and $\mathrm{GeV} \gamma$-ray observations have shown that relativistic jets may exist in $\mathrm{RL}$ NLS1s. ${ }^{1-3}$ As well known, NLS1s are characterized by high accretion rate at sub or super Eddington rate, and the accretion disks can be described by slim model. Considering the important roles played by the magnetic fields in the formation of jets, ${ }^{4}$ a natural conclusion is that large-scale magnetic fields may threading the slim disks. What are the influences of the fields on the dynamics of slim disks? The magnetized slim disk around the neutron star has been studied, ${ }^{5,6}$ however, the magnetized slim disk around black hole is less studied.

\footnotetext{
*This is an Open Access article published by World Scientific Publishing Company. It is distributed under the terms of the Creative Commons Attribution 3.0 (CC-BY) License. Further distribution of this work is permitted, provided the original work is properly cited.
} 


\section{Model}

Following previous work, ${ }^{7}$ the conservation of mass, radial momentum, angular momentum and energy are written as follows,

$$
\begin{aligned}
\frac{d \dot{M}}{d r} & =4 \pi r \dot{m}_{w}, \\
v_{r} \frac{d v_{r}}{d r}+\frac{1}{\Sigma} \frac{d W}{d r} & =\frac{l^{2}-l_{k}^{2}}{r^{3}}-\frac{W}{\Sigma} \frac{d \ln \Omega_{k}}{d r}+g_{m}, \\
\Sigma \frac{v_{r}}{r} \frac{d}{d r}\left(r v_{\phi}\right) & =\frac{1}{r^{2}} \frac{d}{d r}\left(r^{2} \Pi_{r \phi}\right)-\frac{T_{m}}{r}, \\
\int_{-h}^{h} d z \rho T v_{r} \frac{d s}{d r} & =-2 F_{z}+r \Pi_{r \phi} \frac{d}{d r}\left(\frac{v_{\phi}}{r}\right)-2 \dot{m}_{w} \varepsilon,
\end{aligned}
$$

where $r$ is the radius in unit of $r_{g} \equiv 2 G M / c^{2}, v_{r}, v_{\phi}, T, \rho, l, h, l_{k}$ and $\Omega_{k}$ are the radial and azimuthal velocities, temperature, density, angular momentum, height, Keplerian angular momentum and angular velocity, respectively. The quantity $F_{z}$ is the radiative cooling rate per unit surface area, $\dot{M}(r)$ is the accretion rate and $\dot{m}_{w}(r)$ is the mass loss rate per unit area at radius $\mathrm{r}$. The quantities $\Sigma, W$ and $\Pi_{r \phi}$ are the vertical integration of the density, pressure, and $r-\phi$ component of the viscous stress, respectively. For simplicity, the interactions of large-scale magnetic field on the disk are included by three terms, i.e., $g_{m}=B_{r} B_{z} / 2 \pi \Sigma, T_{m}=2 \dot{m}_{w} \Omega(r)\left(r_{A}^{2}-r^{2}\right)$ and $2 \dot{m} \varepsilon=2\left(\gamma_{j}-1\right) \dot{m}_{w} c^{2}$. The field is described by two parameters. The strength at the footpoint is assumed to be a fixed fraction of the total pressure, $\beta$. And along a given field line, the field strength is assumed to be self-similar, $B_{p}(r) \sim B_{p d}\left(r / r_{d}\right)^{-\zeta}$.

\section{Results}

The influences of the magnetic field on the dynamics of the accretion disk are shown in Fig. 1. There are four curves in each panel, which represent the following cases: (i) the case without magnetic field (black long-dashed lines), (ii) the case with the magnetic field of given strength and geometry, i.e. $\beta=0.01, \zeta=2$ (red solid lines), (iii) the case with stronger field, $\beta=0.05$ and $\zeta=2$ (blue short-dashed lines), and (iv) the case with different geometry $\beta=0.01$ and $\zeta=3$ (green dotted lines). From this figure, the following results can be obtained. Firstly, with the existence of the magnetic field, the Mach number is larger, the disk temperature is lower, while the specific angular momentum changes almost ignorably. These are easy to understand. Due to the torque exerted by the magnetic field lines, the angular momentum get removed more efficiently. Therefore the viscous torque reduces and the radial velocity increases. Since the mass loss rate is very small compared to the accretion rate, the specific angular momentum changes only slightly. Secondly, when $\beta$ decreases or $\zeta$ increases, the influences of the field become less significant. This is due to the model, in which the mass loss rate depends on $\beta$ and $\zeta$ as shown in Equation 8. Thirdly, the mass loss due to the jet are dominated by the inner region. 

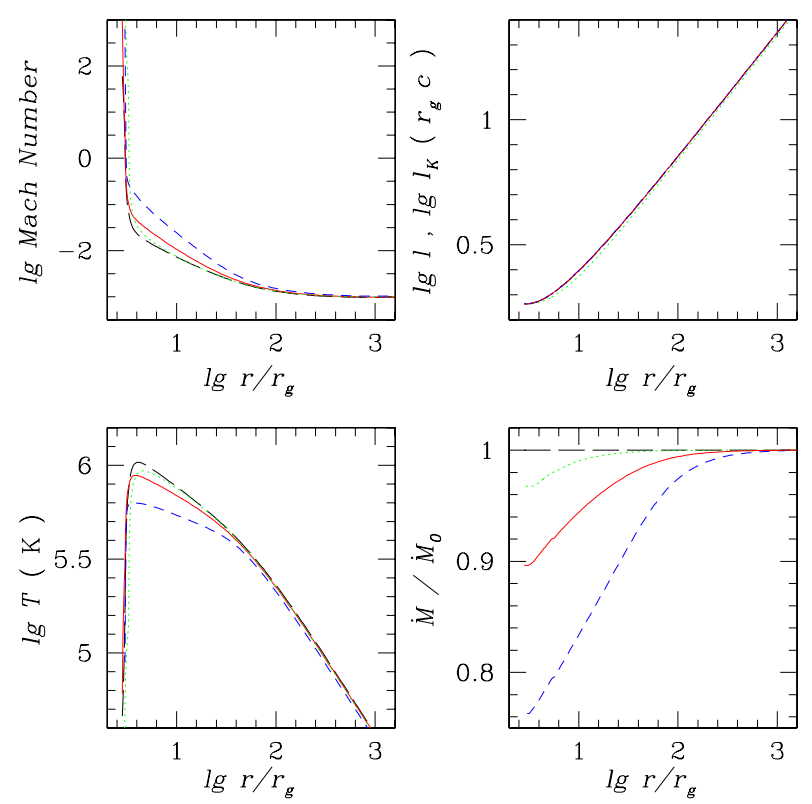

Fig. 1. Dynamical effects of the magnetic field for $\alpha=0.3$ and $\dot{M}_{0}=L_{E d d} / c^{2}$. The long dashed line corresponds to the case without magnetic field, the solid line corresponds to $\beta=0.01$ and $\zeta=2$, the short dashed line corresponds to $\beta=0.05$ and $\zeta=2$, and the dotted line corresponds to $\beta=0.01$ and $\zeta=3$. In the upper right panel, the Keplerian angular momentum is also shown, which is almost the same as the angular momentum of the disk without magnetic field.

For smaller viscous parameter $\alpha$, the influence of the magnetic field is stronger. This is because the relative contribution of the magnetic torque on the disk is larger since the viscous torque is smaller. In contrast, for higher accretion rate, the influence of the magnetic field is much less significant. This is because the angular velocity of the disk decreases with the accretion rate, and the corresponding Alfvénic radius increases, which means the outflow gas would be more efficient in taking away angular momentum. Therefore, the mass loss rate decreases.

The ratio between the kinetic power of jet and the disk luminosity is also calculated as shown in Fig. 2. At lower accretion rate the ratio decreases with accretion rate, while at high accretion rate the ratio increases with accretion rate. Since the jet power is proportional to $\dot{M}_{\text {jet }}$, which changes monotonically with accretion rate, the variation of $P_{\text {jet }} / L_{\text {disk }}$ is mainly due to photon trapping. At lower accretion rate, photon trapping is not significant, and the disk luminosity is proportional to accretion rate. But at higher accretion rate, the disk is optically thick enough that photon trapping becomes important, and the disk luminosity gets saturate [28]. Therefore, at lower accretion rate the jet kinetic power increases slower than the disk luminosity, while at higher accretion rate, the jet kinetic power increases faster than the disk luminosity. 


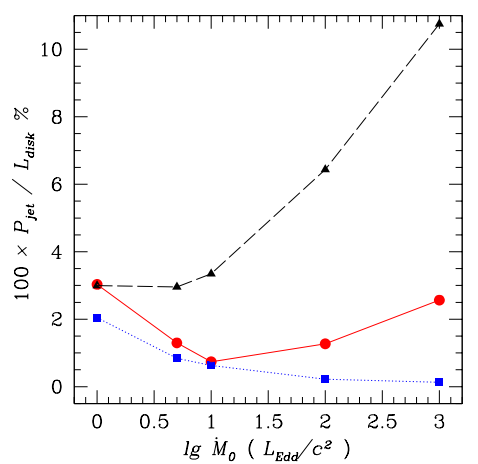

Fig. 2. The ratio of jet kinetic power to disk luminosity with $\alpha=0.3$. The solid line corresponds to $\beta=0.01$ and $\zeta=2$, the dashed line corresponds to $\beta=0.05$ and $\zeta=2$, and the dotted line corresponds to $\beta=0.01$ and $\zeta=3$.

It can also be seen from Fig. 2 that the intrinsic jet power is only a small fraction of the disk luminosity. This result support the argument that RL NLS1 are similar to blazars, in which the jet axis is aligned to the eyesight. If the outflow due to radiation pressure is included, this result can still hold except the case when the radiation is so strong that most gas is pushed away rather than accreted to the $\mathrm{BH}$.

\section{Acknowledgments}

This work is supported in part by the Natural Science Foundation of China (grant 11333004, 11233006) and the Natural Science Foundation of Fujian Province (No. 2011J01023).

\section{References}

1. H.-Y. Zhou et al., Astrophys. J. 584, 147 (2003).

2. W. Yuan et al., Astrophys. J. 685, 801 (2008).

3. F. D'Ammando et al., arXiv:1309.5366.

4. D. L. Meier, S. Koide and Y. Uchida, Science 291, 84 (2001).

5. D. Lai, Astrophys. J. 502, 721 (1998).

6. U. Lee, Astrophys. J. 511, 359 (1999).

7. S.-L. Li and X. Cao, Mon. Not. R. Astron. Soc. 4001734 (2009). 